\title{
The Effect of Fibrin-Stabilizing Factor on the Subunit Structure of Human Fibrin
}

\author{
Martin L. Schwartz, Salvatore V. Pizzo, Robert L. Hill, and \\ Patrick A. McKeE \\ From the Department of Biochemistry, Duke University Medical Center ana \\ the Department of Medicine, Veterans Administration Hospital, \\ Durham, North Carolina 27706
}

\begin{abstract}
A в S TRACT The formation of human fibrin from fibrinogen has been examined by polyacrylamide gel electrophoresis in sodium dodecyl sulfate, a method which separates a mixture of proteins on the basis of differences in molecular weight. It has been found that the plasma from a patient with a congenital deficiency of fibrin-stabilizing factor forms clots lacking the cross links among the $\alpha$ - and $\gamma$-chains found in normal, crosslinked human fibrin. The addition of purified fibrinstabilizing factor or normal plasma to the deficient plasma results in extensive cross-linking of the chains. Thus, the fibrinogen in the fibrin-stabilizing factor deficient plasma appears to be normal and forms fibrin which contains dimeric, cross-linked $\gamma$-chains and polymeric, high molecular weight forms of $\alpha$-chains. By the use of these electrophoretic methods, it has also been possible to develop a highly sensitive method for measuring the content of fibrin-stabilizing factor in plasma. This method depends upon the use of urea-treated fibrinogen, which is completely devoid of fibrin-stabilizing factor, but which forms the usual cross-linked subunits after conversion to fibrin by thrombin in the presence of fibrin-stabilizing factor.
\end{abstract}

\section{INTRODUCTION}

In 1944, Robbins observed that a serum factor promoted the formation of fibrin which was insoluble in di-

Preliminary reports of this work were given at The American Society for Clinical Investigation, Atlantic City, N. J., 1970 and the Central Society for Clinical Research, Chicago, Ill., 1970.

Mr. Schwartz and Mr. Pizzo are predoctoral Fellows, Medical Scientist Training Program, National Institute of General Medical Sciences, National Institutes of Health (5T05 GM 01678-05).

Received for publication 14 December 1970 and in revised form 3 February 1971. lute acids and alkalis (1). Four years later, Laki and Lorand confirmed this finding and introduced the use of $5 \mathrm{M}$ urea as a solubilizing agent (2). Subsequently this factor was named fibrin-stabilizing factor (FSF). ${ }^{1}$ Loewy, Dunathan, Gallant, and Gardner demonstrated that the action of FSF was enzymatic (3) ; Lorand and Konishi showed that an inactive precurser of FSF existed in plasma which could be activated by thrombin and calcium (4). The inactive precursor has been officially recognized and named factor XIII. Recently, FSF has been shown to catalyze a transpeptidation reaction between lysine and glutamine residues, which leads to the formation of $\epsilon$-( $\gamma$-glutamyl) lysine cross-links between fibrin monomers to produce urea-insoluble fibrin (5-8).

We previously reported that human fibrinogen and noncross-linked, urea-soluble fibrin contain three pairs of subunit polypeptide chains, the $\alpha_{-}, \beta$ - , and $\gamma$-chains, ${ }^{2}$ with molecular weights of $73,000,60,000$, and 50,000 respectively $(9,10)$. In contrast, urea-insoluble human fibrin was found to contain cross-linked $\gamma$-chains of molecular weight 105,000, and cross-linked $\alpha$-chains of molecular weight greater than 400,000 (10). The formation of cross-links in the $\alpha$ - and $\gamma$-chains to produce the so-called $\gamma$-dimer and $\alpha$-polymer subunits of ureainsoluble fibrin is presumably the result of the introduction of $\epsilon$-( $\gamma$-glutamyl) lysine cross-links by FSF. The $\beta$-chains do not appear to be involved in the cross-linking by FSF. Thus, highly cross-linked fibrin contains crosslinks between two $\gamma$-chains and cross-links among several $\alpha$-chains, although present methods cannot ex-

${ }^{1}$ Abbreviations used in this paper: FSF, fibrin-stabilizing factor; NEM, N-ethylmaleimide; PCMB, p-chloromercuribenzoate; SDS, sodium dodecyl sulphate.

2 Throughout this text the subunits of fibrinogen and fibrin will be designated as $\alpha, \beta$, and $\gamma$ in contrast to the designation of $\alpha(\mathrm{A}), \beta(\mathrm{B})$, and $\gamma$ which is often used for fibrinogen. 
clude unequivocally the possibility of very small amounts of cross-links between $\alpha$ - and $\gamma$-chains.

We wish to report here further studies on the crosslinking of human fibrin by FSF, including a description of a highly sensitive method for the detection of FSF in human plasma. It has been found that fibrin from a patient with FSF deficiency contains neither $\boldsymbol{\gamma}$-dimer, $\alpha$-polymer, nor chains with abnormal crosslinks. When an exogenous source of FSF is added to the deficient plasma, normal cross-linking occurs. Furthermore, it has been found that electrophoresis on polyacrylamide gels in sodium dodecyl sulfate can be applied not only to examine the subunit structure of fibrinogen and fibrin as reported earlier (10), but also specifically to detect small amounts of FSF in plasma when urea-treated fibrinogen is used as a substrate. These techniques make it possible to examine a patient's fibrin formed from whole plasma without the necessity of purifying the patient's fibrinogen. This methodology is extremely simple and may be adopted readily by any laboratory which is equipped to run polyacrylamide gels.

\section{METHODS}

Reagents. Analytical grade reagents were used throughout unless stated otherwise. A stock solution of bovine thrombin (Upjohn Co., Kalamazoo, Mich.; $13 \mathrm{NIH} \mathrm{U/}$ absorbancy unit at $280 \mathrm{~nm}$ ) was prepared by dissolving $1000 \mathrm{U}$ in $2 \mathrm{ml}$ of glycerol: $0.15 \mathrm{M}$ potassium chloride $(60: 40, v: v)$ and stored at $-20^{\circ} \mathrm{C}$ for up to $3 \mathrm{wk}$. Prior to use, the stock solution was diluted to $50 \mathrm{U}$ per $\mathrm{ml}$ with $0.15 \mathrm{M}$ potassium chloride containing $0.2 \mathrm{M}$ calcium chloride. This is the thrombin-calcium solution referred to throughout the text when fibrinogen is converted to fibrin. Factor XIII was purified using Kazama and Langdell's (11) modifications of the method of Loewy, Dunathan, Kriel, and Wolfinger (12). The specific activity of the material prepared by us was 6000 -fold greater than that of pooled normal plasma when tested by the method of Loewy et al. (12) on urea-treated fibrinogen.

Fibrinogen purification and inactivation of FSF. Purified normal human fibrinogen ( $97 \%$ clottable) was prepared by the method of Blomback and Blomback (13) from fresh frozen plasma. Fibrinogen, purified by this method through the I-4 step, contained a substantial amount of FSF activity. Because such fibrinogen preparations were unsuitable as a substrate in FSF assays, two different methods (14-16), which have been described earlier, were used to inactivate FSF in fibrinogen. These were performed as follows: (a) Human fibrinogen (fraction I-4 of Blomback [13]) was dissolved in $0.15 \mathrm{M}$ ammonium acetate buffer, $\mathrm{pH} 7.4$, to a final concentration of $0.5 \%$ and mixed with an equal volume of $0.01 \mathrm{M} p$-chloromercuribenzoate (PCMB) which was prepared by diluting with water from a stock solution of $0.6 \mathrm{M}$ PCMB in $0.05 \mathrm{M}$ potassium hydroxide. The mixture was stirred at room temperature in the dark for $1 \mathrm{hr}$ and then dialyzed for $24 \mathrm{hr}$ at $4^{\circ} \mathrm{C}$ against four changes in $150 \mathrm{vol}$ of 0.15 M ammonium acetate buffer, $(\mathrm{pH} 7.4) .1 \mathrm{ml}$ portions were stored at $-20^{\circ} \mathrm{C}$. (b) Lyophilized human fibrinogen (fraction I-4 of Blomback (13)) was dissolved in $3.3 \mathrm{M}$ urea in $0.1 \mathrm{M}$ sodium phosphate buffer, $\mathrm{pH} 7.4$, to give a final concentration of $2 \mathrm{mg} / \mathrm{ml}$. This solution was kept at room temperature without stirring for $12 \mathrm{hr}$ and then dialyzed twice for $4 \mathrm{hr}$ against $1000 \mathrm{vol}$ of $0.3 \mathrm{M} \mathrm{NaCl}$. The fibrinogen solution was then divided into portions and stored frozen at $-20^{\circ} \mathrm{C}$.

FSF-deficient plasma. This plasma was from a patient congenitally deficient in FSF who was studied originally by Barry and Delage (17). We wish to thank Dr. R. G. Mason (Department of Pathology, University of North Carolina) for the generous gift of this plasma.

Normal human plasma. Nine parts of blood were drawn by venepuncture into one part of $3.5 \%$ sodium citrate and then centrifuged at $3500 \times g$ at $4^{\circ} \mathrm{C}$ for $30 \mathrm{~min}$. The plasma was stored in portions at $-20^{\circ} \mathrm{C}$.

Fibrin formation. Unless stated otherwise, fibrin was formed as follows: A thrombin-calcium solution $(0.01 \mathrm{ml})$ was added to $0.1 \mathrm{ml}$ plasma or fibrinogen solution $(2 \mathrm{mg} /$ $\mathrm{ml}, 0.15 \mathrm{M}$ ammonium acetate buffer, $\mathrm{pH}$ 7.4) contained in $13 \times 75 \mathrm{~mm}$ test tubes. If exogenous FSF was to be included in the clotting mixture, it was added to the fibrinogen solution before the addition of the thrombin-calcium mixture. Clotting was always visible in either plasma or fibrinogen solutions within 5-10 sec after addition of the thrombin-calcium mixture. In those experiments where plasma was clotted by addition of calcium, visible clotting occurred within 4-6 min. The fibrin clots were allowed to form at room temperature for $2 \mathrm{hr}$ unless indicated otherwise. The tube containing the fibrin was then filled with $0.15 \mathrm{M} \mathrm{NaCl}$, and the fibrin removed with a metal spatula and blotted on Whatman No. 1 filter paper to remove as much fluid as possible. The fibrin was then dissolved in 0.3 $\mathrm{ml}$ solution of $9 \mathrm{M}$ urea containing $3 \%$ sodium dodecyl sulfate and $3 \%$ mercaptoethanol in $0.04 \mathrm{M}$ sodium phosphate buffer, $\mathrm{pH}$ 7.1. The fibrin dissolved in this denaturing solution within $12 \mathrm{hr}$ at $37^{\circ} \mathrm{C}$ and the final fibrin concentration in the resulting solution was $0.5-0.7 \mathrm{mg} / \mathrm{ml}$. Portions $(0.015$ $\mathrm{ml}$ ) of this solution were added to $0.005 \mathrm{ml}$ of $0.05 \%$ bromophenol blue and subjected to electrophoresis as described below. It should be noted that all disulfide bonds in fibrinogen and fibrin were reduced under these conditions and remained so during electrophoresis as evidenced by the fact that fully reduced and carboxymethylated fibrinogen and fibrin gave electrophoretic patterns identical with those obtained under the above conditions.

Solubility assays. $2 \%$ acetic acid, $6 \mathrm{~m}$ urea, and $6 \mathrm{M}$ guanidine hydrochloride were used as dispersing media in assays for insoluble fibrin formation. The $2 \%$ acetic acid proved satisfactory for routine use and was added in the arbitrary ratio of $1 \mathrm{ml}$ for each $0.1 \mathrm{mg}$ of fibrin. Fibrin solubility was then observed for $18 \mathrm{hr}$, although in accord with Tyler (18), no difference in solubility was found on incubation beyond $1 \mathrm{hr}$.

Electrophoresis. The technique of polyacrylamide gel electrophoresis in sodium dodecyl sulfate (SDS) was similar to techniques described earlier $(19,20)$. The application of this method to the structural studies of both fibrinogen and fibrin has been previously reported $(10,21)$. It should be noted that in the present study both $5 \%$ and $7.5 \%$ polyacrylamide gels were used. Molecular weight determinations in the range of $47,000-100,000$ were performed on $5 \%$ gels according to the method of Weber and Osborn (19). However, it was found that sharper resolution of protein bands could be obtained by using $7.5 \%$ gels prepared as usual except that the final polymerizing solution contained $4 \%$ of $0.5 \mathrm{M} \mathrm{EDTA}$ and $60 \%$ of $10 \mathrm{M}$ urea. Both the $5 \%$ and $7.5 \%$ gels were allowed to polymerize for at least $1 \mathrm{hr}$ before use. The electrophoresis buffer consisted of $0.1 \%$ SDS 


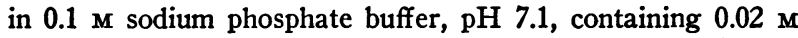
EDTA. All electrophoreses were performed at $20^{\circ}-25^{\circ} \mathrm{C}$ at a constant current of 5 ma per tube until the bromophenol blue marker dye had migrated to about $1 \mathrm{~cm}$ of the gel bottom. The gels were stained with Coomassie blue and destained in acetic acid: methanol: distilled water as previously reported $(10)$.

The amounts of each of the protein bands on the gels were measured by scanning the destained gels in a Gilford Linear Transport system (Gilford Instrument Co., Oberlin, Ohio) connected to a Beckman DU recording spectrophotometer (Beckman Instruments, Inc., Fullerton, Calif.). The areas under each peak were determined by planimetry.

\section{RESULTS}

Fibrin from normal plasma. Fig. 1 shows the SDSgel electrophoretic patterns of reduced purified human fibrinogen (Fig. 1 $a$ ), noncross-linked fibrin (Fig. $1 b$ ), partially cross-linked fibrin (Fig. $1 c$ ), and cross-linked normal fibrin (Fig. $1 d$ ). The reduced, completely noncross-linked soluble fibrin (Fig. $1 b$ ) was formed by clotting $0.1 \mathrm{ml}$ of normal plasma in the presence of $0.1 \mathrm{ml}$ of $0.5 \mathrm{M}$ EDTA with $0.5 \mathrm{U}$ of thrombin. The three major bands in soluble fibrin correspond to the $\alpha$-, $\beta$-, and $\gamma$-chains of purified fibrinogen. The $\alpha$-chain in both fibrinogen and noncross-linked fibrin has a major component and two minor components, in accord with our earlier findings and those of other investigators $(10,21,22)$.

Incompletely cross-linked fibrin is shown for comparison in Fig. $1 \mathrm{c}$. This fibrin was formed by mixing $0.01 \mathrm{ml}$ of a thrombin-calcium solution with $0.1 \mathrm{ml}$ of normal plasma, and after $40 \mathrm{sec}$, suspending the clot in $3 \mathrm{ml}$ of $0.15 \mathrm{M} \mathrm{NaCl}$. After washing for $20-30 \mathrm{sec}$ in the $\mathrm{NaCl}$, the fibrin was blotted and dissolved in a solution of $9 \mathrm{~m}$ urea, containing $3 \%$ sodium dodecyl sulfate and $3 \%$ mercaptoethanol in $0.04 \mathrm{M}$ sodium phosphate buffer, $\mathrm{pH}$ 7.1. Compared to noncross-linked fibrin, the $\alpha$-chains are present in smaller amounts whereas the $\beta$-chain is unchanged. The $\gamma$-chain has disappeared and a new band of somewhat lower mobility than $\alpha$-chain has appeared. This new band has been shown to be the $\gamma$-dimer, which is formed on cross-linking two $\gamma$-chains. Several faint, but distinct species of higher molecular weight than the $\gamma$-dimer are also noted. Fig. $1 d$ shows the pattern for reduced, completely cross-linked fibrin which was formed by mixing $0.01 \mathrm{ml}$ of a thrombincalcium solution with $0.1 \mathrm{ml}$ of normal plasma and incubating the mixture for $2 \mathrm{hr}$. The three darkest staining bands observed have been shown to be the $\beta$ monomer, the $\gamma$-dimer, and the polymer of $\alpha$-chain ( $\alpha$-polymer), which remains at the top of the gel (10). A less intensely staining band of mobility intermediate between that of the $\alpha$-chain and $\beta$-chain is also noted. Presumably this band is derived from the $\alpha$-chain but its exact relationship to the minor $\alpha$-chain components remains unclear. Several faint, but distinct bands are noted with a mobility greater than that of $\alpha$-polymer but less than that of $\gamma$-dimer, just as in partially cross-linked fibrin.

Fibrin from FSF-deficient plasma. Fibrin was formed by incubating $0.1 \mathrm{ml}$ of a FSF-deficient plasma with $0.1 \mathrm{ml}$ of $0.5 \mathrm{~m}$ EDTA containing $0.5 \mathrm{U}$ of thrombin

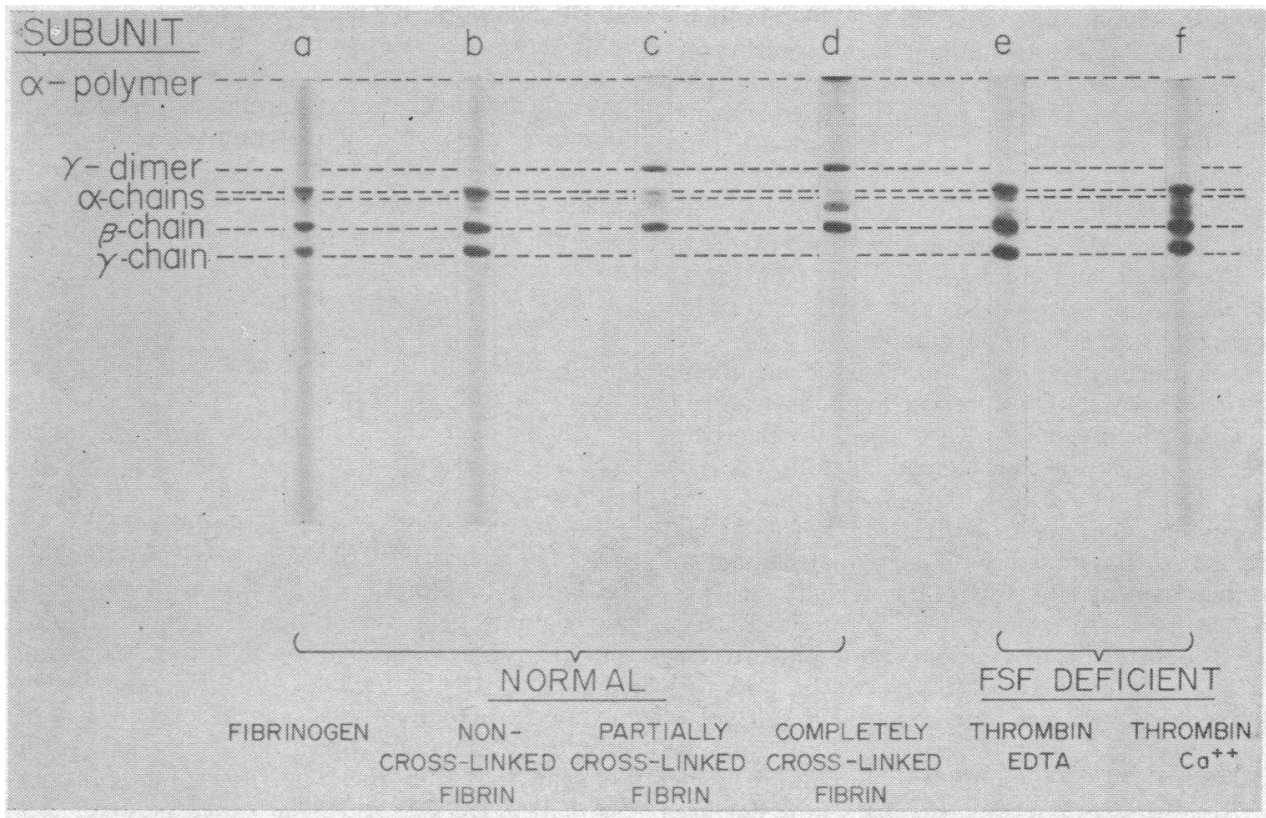

FIGURE 1 Electrophoretic patterns of reduced purified fibrinogen and reduced fibrin from normal and FSF-deficient plasma on $7.5 \%$ polyacrylamide gels in sodium dodecyl sulfate. 


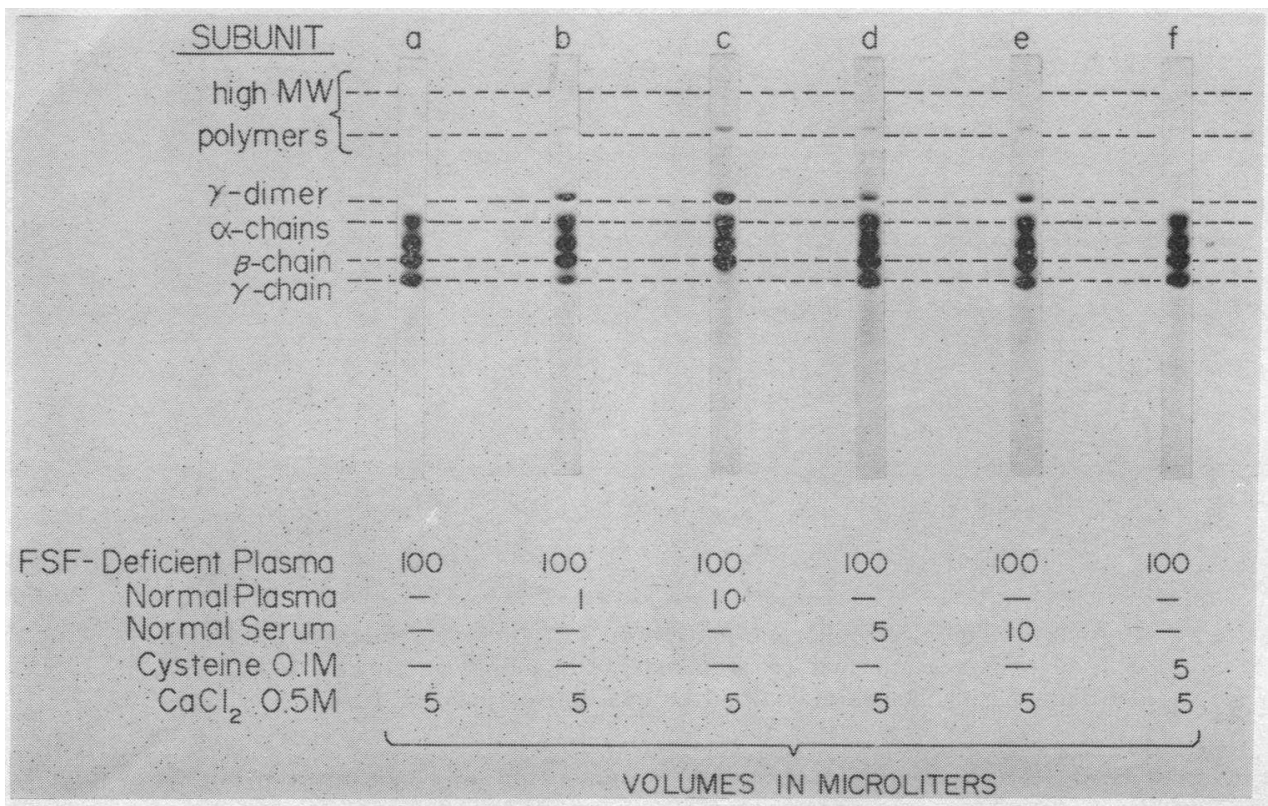

FIGURE 2 The effect of normal plasma, normal serum, and cysteine during fibrin formation in FSF-deficient plasma as seen on $5 \%$ polyacrylamide gel electrophoresis in sodium dodecyl sulfate.

for $2 \mathrm{hr}$ at room temperature. The fibrin was then reduced with $3 \%$ mercaptoethanol containing $9 \mathrm{M}$ urea and $3 \%$ sodium dodecyl sulfate in $0.04 \mathrm{~m}$ phosphate buffer, $\mathrm{pH} 7.1$, and analyzed by gel electrophoresis. Fig. $1 e$ shows that the electrophoretic pattern is essentially identical with that given by reduced, normal noncrosslinked fibrin (Fig. $1 b$ ). Fig. $1 f$ shows the pattern for fibrin prepared by allowing $0.1 \mathrm{ml}$ of FSF-deficient plasma to clot for $2 \mathrm{hr}$ after mixing with $0.1 \mathrm{ml}$ of a thrombin-calcium solution. The mobilities of the $\alpha-, \beta-$ and $\gamma$-bands are unchanged from those shown in Fig. $1 e$. Except for a barely visible band in the $\gamma$-dimer region and three very faint higher molecular weight bands there is no evidence of cross-linking. It must be emphasied that these trace amounts of higher molecular weight species were not caused by FSF-contamination of thrombin because similar bands were observed after recalcification only. It should be noted that the $\alpha$ region in Fig. $1 e$ shows a major and two very faint minor bands. In Fig. $1 f$, however, a darkly staining band is noted with a mobility between that of $\alpha$-chain and $\beta$-chain. This band may be identical with the band of a similar mobility noted in completely cross-linked fibrin (Fig. $1 d)$.

The fibrinogen in the FSF-deficient plasma could be cross-linked by the addition of either normal plasma or serum, as shown in Fig. 2. Fig. $2 a$ shows the pattern for reduced fibrin formed by recalcifying FSF-deficient plasma. Figs. $2 b-2 e$ show that small amounts of normal plasma or serum provided sufficient amounts of
FSF to form the $\gamma$-dimer and traces of high molecular weight polymers in the fibrin formed in FSF-deficient plasma. Although the amount of cross-linking clearly increased with the quantity of normal plasma or serum added before clotting the FSF-deficient plasma, it should be noted that $0.1 \mathrm{ml}$ of normal serum caused less crosslinking than $0.01 \mathrm{ml}$ of normal plasma. Fig. $2 f$ shows that cysteine, which increases FSF reactivity, did not cause cross-linking when added before recalcifying the FSF-deficient plasma. Fig. 3 illustrates that the addition of as little as $4 \mu \mathrm{g}$ of highly purified FSF to 100 $\mu 1$ of FSF-deficient plasma resulted in a pattern of cross-linking indistinguishable from that of fibrin from normal plasma after $2 \mathrm{hr}$.

Inactivation of FSF by p-chloromercuribenzoate $(P C M B)$ and $N$-ethylmaleimide $(N E M)$. The fibrinogen in fraction I-4 of Blomback (13) contains varying but significant amounts of FSF, since the fibrin formed from this fibrinogen is usually insoluble in dilute acetic acid or urea and shows extensive cross-linking on SDSgel electrophoresis (Fig. $4 a$ ).

Because FSF is inactivated by sulfhydryl reagents $(3,16)$, and sulfhydryl groups are absent in fibrinogen (23), Swigert, Koppel, and Oliver proposed using PCMB-treated fibrinogen as the substrate in assays for FSF (14). When purified fibrinogen (fraction I-4) that had been reacted with PCMB was clotted by thrombincalcium for $2 \mathrm{hr}$, the fibrin was completely soluble in $2 \%$ acetic acid within $5 \mathrm{~min}$. If solubility was the sole criterion, the Swigert method of testing for the pres- 


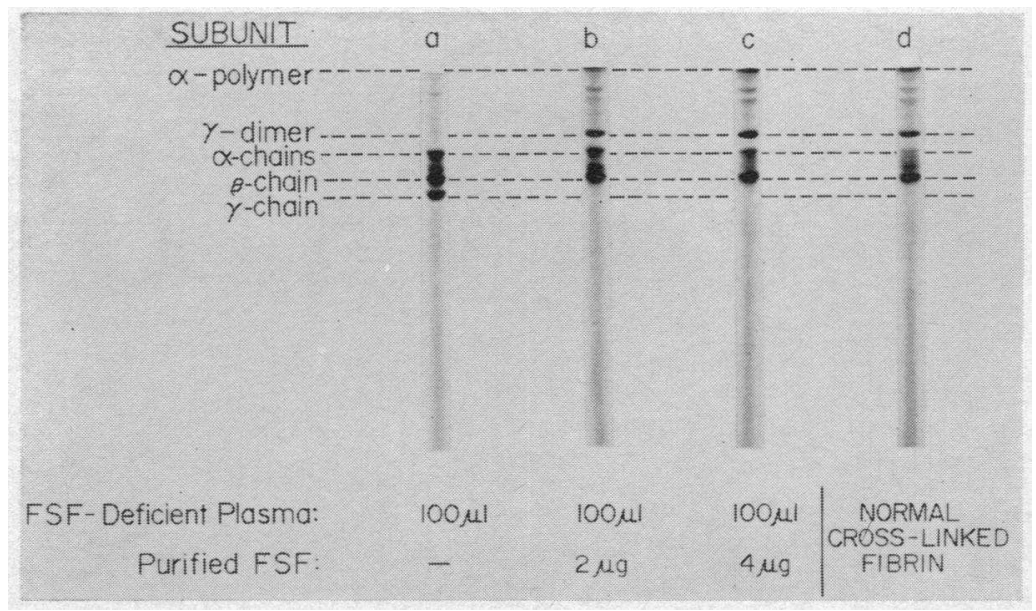

FIGURE 3 The electrophoretic pattern on 7.5 polyacrylamide gels in sodium dodecyl sulfate of normal cross-linked fibrin and fibrin formed from FSFdeficient plasma before and after the addition of purified FSF.

ence or absence of FSF activity appeared satisfactory, since only soluble fibrin was formed when FSF-deficient plasma was added to the fibrinogen treated with PCMB, and insoluble fibrin formed when normal plasma was added. SDS-gel electrophoresis showed, however, that fibrin from PCMB-treated fibrinogen in the presence of either normal or FSF-deficient plasma contained significant amounts of $\boldsymbol{\gamma}$-dimer and higher molecular weight polymers. As expected, the cross-linking was more complete when normal plasma was used. This is most likely explained by reversal of the PCMB inactivation of FSF by the large number of free thiol groups in normal or FSF-deficient plasma (24). It was impossible to remove substances containing sulfhydryl groups, from normal and FSF-deficient plasma by dialysis, since dialyzed preparations caused cross-linking, although to a lesser extent than before dialysis. Further- more, the supernatant solution from boiled, normal, and FSF-deficient plasma still caused small amounts of $\gamma$-dimer formation. Finally, when cysteine alone was added before clotting the fibrinogen, which had been treated with PCMB, insoluble, cross-linked fibrin was formed readily.

Because sulfhydryl groups can be regenerated from fibrinogen treated with PCMB, N-ethylmaleimide (NEM), which reacts irreversibly with thiol groups, was tested as a means for inactivation of the FSF in fibrinogen preparations (25). A solution of purified fibrinogen (fraction $\mathrm{I}-4 ; 5 \mathrm{mg} / \mathrm{ml}$ ) was incubated for $2 \mathrm{hr}$ with $0.2 \mathrm{M} \mathrm{NEM}$ in $0.1 \mathrm{M}$ sodium phosphate buffer, $\mathrm{pH}$ 7.0. The reaction mixture was then dialyzed against $0.05 \mathrm{~m}$ Tris buffer, $\mathrm{pH} 7.4$, containing $0.1 \mathrm{~m}$ sodium chloride to remove excess NEM, and clotted by mixing with a thrombin-calcium solution for $2 \mathrm{hr}$. Electropho-

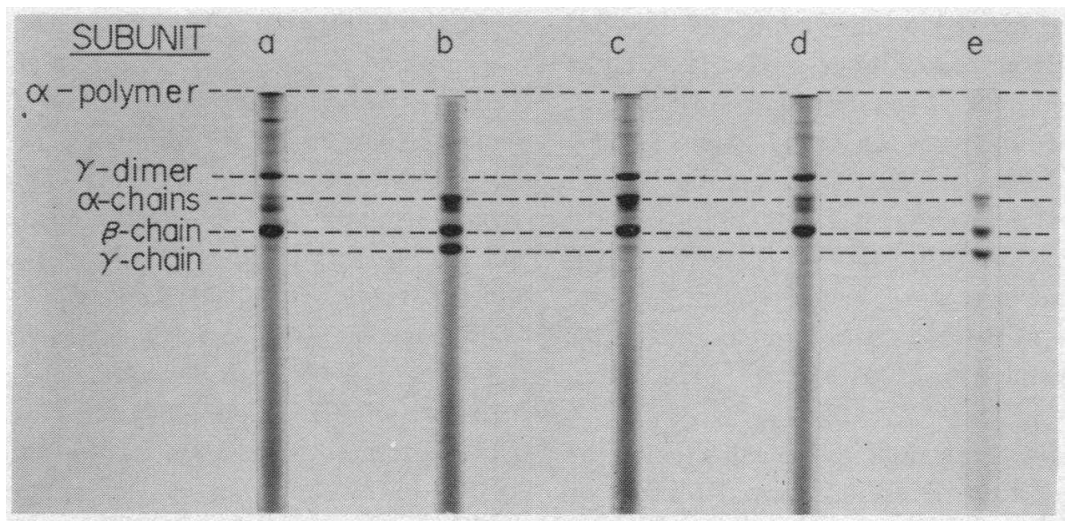

FIgURE 4 Electrophoretic patterns of fibrin from: (a) Blomback I-4 fibrinogen; (b) Blomback I-4 fibrinogen after exposure to $3.3 \mathrm{M}$ urea; (c) ureatreated fibrinogen plus normal plasma; $(d)$ urea-treated fibrinogen plus purified FSF; and $(e)$ urea-treated fibrinogen plus FSF-deficient plasma. 


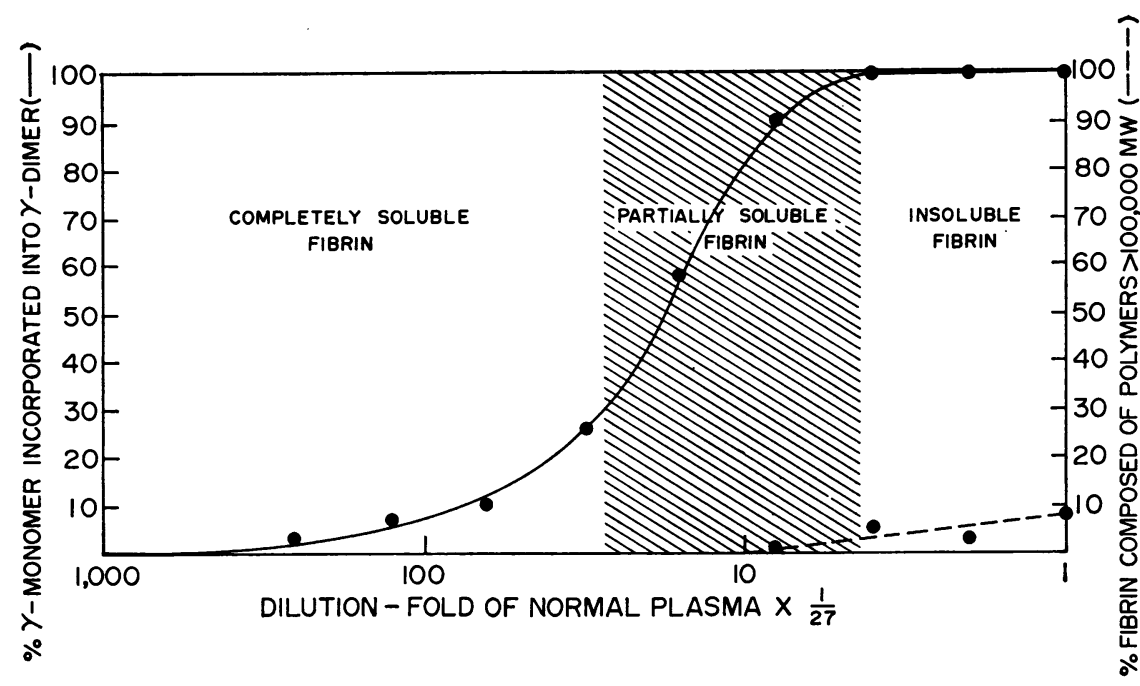

FIGURE 5 The relationship between the solubility of fibrin in $2 \%$ acetic acid and the content of $\gamma$-dimer and high molecular weight polymers.

resis of the reduced fibrin showed that complete crosslinking had occurred. When $0.2 \mathrm{ml}$ of $0.3 \mathrm{~m} \mathrm{NEM}$ in 0.1 M sodium phosphate buffer $\mathrm{pH} 7$ was mixed with $0.1 \mathrm{ml}$ of fibrinogen (fraction I-4) or normal plasma and clot formation initiated by reacting with thrombin-calcium solution for $2 \mathrm{hr}$, no evidence of cross-linking was noted on electrophoresis of the fibrin formed. These results suggest that FSF as found in plasma may be inactivated by PCMB but not NEM; however, after FSF is activated by thrombin (4), it may be inactivated by NEM. These results with NEM are essentially identical with those reported for iodoacetate (3). Thus, a more suitable method for inactivating FSF was sought that would be applicable to the highly sensitive SDS-gel electrophoretic technique.

Inactivation of FSF by urea. When urea-treated fibrinogen was reacted with thrombin, fibrin began forming within $10 \mathrm{sec}$. The fibrin formed after $2 \mathrm{hr}$ dissolved within $20 \mathrm{sec}$ in 10 times its volume of $2 \%$ acetic acid. Electrophoresis of this fibrin showed only $\alpha-, \beta-$, and $\gamma$-chains and a slight trace of a band in the $\gamma$-dimer region (Fig. $4 b$ ). When a mixture of $0.01 \mathrm{ml}$ of normal plasma and $0.1 \mathrm{ml}$ of urea-treated fibrinogen $(2 \mathrm{mg} / \mathrm{ml})$ was reacted with a thrombin-calcium solution for $2 \mathrm{hr}$, the resulting fibrin was devoid of $\gamma$-monomer which had been completely converted to the $\gamma$-dimer (Fig. $4 c$ ). The addition of $2 \mu \mathrm{g}$ of FSF to $200 \mu \mathrm{g}$ of urea-treated fibrinogen resulted in extensive cross-linking (Fig. $4 d$ ). However, when FSF-deficient plasma was added to the urea-treated fibrinogen, no cross-linking occurred (Fig. $4 e$ ).

Fig. 5 shows the relationship between the solubility of fibrin and its content of $\gamma$-dimer and high molecular weight polymers. In these experiments, serial dilutions of normal plasma were added to a constant volume of urea-treated fibrinogen $(1.5 \mathrm{mg} / \mathrm{ml})$ so that the final concentration of FSF ranged from $1 / 27$ to $1 / 6912$ that of normal plasma. Each of these mixtures was clotted by the thrombin-calcium solution for $2 \mathrm{hr}$, and the resulting clot reduced and analyzed electrophoretically. The stained gels were scanned and the area beneath each peak quantitated by planimetry. The extent of $\boldsymbol{\gamma}$-dimer formation was expressed as a percentage of the total area under the peaks corresponding to $\gamma$-chain plus the $\gamma$-dimer. Fibrin containing $26 \%$ or less of the $\gamma$-chain in the form of $\gamma$-dimer was completely soluble in $2 \%$ acetic acid within $1 \mathrm{hr}$. When $58 \%$ of the $\gamma$-chain had been cross-linked to give the $\gamma$-dimer, the fibrin was no longer completely soluble. From these data it was estimated that the amount of FSF in approximately $0.01 \mathrm{ml}$ of plasma will cause $100 \%$ dimerization of the $\gamma$-chains in $1.0 \mathrm{mg}$ of fibrinogen in $2 \mathrm{hr}$. Fig. 5 also indicates that 64 times the amount of FSF, necesary to demonstrate $\gamma$-dimer formation by SDS-gel electrophoresis, is required to produce fibrin which is completely insoluble in $2 \%$ acetic acid over $18 \mathrm{hr}$. Finally, it should be noted that the appearance of high molecular weight polymers coincides with the end of $\gamma$-dimer formation and the onset of complete fibrin insolubility.

\section{DISCUSSION}

Urea and acetic acid solubility tests have not eliminated the possibility that fibrin from FSF-deficient patients is partially or abnormally cross-linked. By the electrophoretic analyses described here, it is clear that neither $\gamma$-dimer, $\alpha$-polymer, nor abnormal polymer formation oc- 
cur in fibrin formed in FSF-deficient plasma. However, if normal plasma, normal serum, or highly purified FSF is added to the FSF-deficient plasma, both $\gamma$-dimerization and $\alpha$-polymerization proceed at rates similar to those for normal fibrin formation. Under the experimental conditions described here, the $\gamma$-chains are crosslinked completely during the first minute or two of fibrin formation whereas the $\alpha$-chain is cross-linked during the next $1-2 \mathrm{hr}$.

Treatment of fibrinogen with urea proved to be an excellent means for inactivating the FSF which contaminates most fibrinogen preparations. It should be noted that fibrinogen used in these studies was prepared from fresh-frozen plasma. Presumably, FSF is selectively and irreversibly denatured by exposure to $3.3 \mathrm{M}$ urea while the tertiary structure of fibrinogen is sufficiently unaffected so that $\gamma$-chain as well as $\alpha$-chain cross-links can form in response to exogenous FSF. The polypeptide chains in urea-treated fibrinogen do not cross-link when treated with thrombin and calcium in the presence or absence of cysteine, and the fibrin formed dissolves in $2 \%$ acetic acid within $20 \mathrm{sec}$. If a small amount of normal plasma, normal serum, or purified FSF was added to urea-treated fibrinogen, the resulting fibrin after reaction with thrombin is not only insoluble in $2 \%$ acetic acid but also shows $\gamma$ - and $\alpha$-chain crosslinks.

It is to be emphasized that treatment of fibrinogen with PCMB is an unsatisfactory method for preparing fibrinogen completely free of FSF activity, because PCMB inactivation is easily reversed by extraneous sulfhydryl groups. For this reason PCMB-treated fibrinogen is not the optimal substrate when assaying plasma or tissue fluid since reactivation of PCMB-inactivated FSF by sulfhydryl groups could be mistaken for low levels of FSF activity (26).

The studies described here also reinforce previous notions that minute amounts of FSF cross-link large quantities of fibrinogen $(3,27,28)$. Experiments in which urea-treated fibrinogen served as substrate, suggest that when the plasma FSF level is as little as $1 \%$ of normal, the cross-linkage of $\gamma$-chains is complete within $2 \mathrm{hr}$ although the cross-linkage of $\alpha$-chains is incomplete. However, fibrin formed under these conditions is completely insoluble in acetic acid and urea when judged visually at $18 \mathrm{hr}$. The data in Fig. 5 also show that completely soluble fibrin may still contain a significant amount of cross-linked $\gamma$-chains and that completely insoluble fibrin may contain very low amounts of cross-linked $\alpha$-chains. It is important to emphasize that despite this situation, the usual observation of fibrin solubility in $2 \%$ acetic acid or $5 \mathrm{~m}$ urea is a satisfactory screening method for detecting FSF deficiency. It must be kept in mind, however, that completely insoluble clots may be formed in plasma with very low levels of FSF. Thus, the only method for demonstrating FSF levels lower than those detected by solubility assays is fibrin analysis on SDS-polyacrylamide gel electrophoresis.

Fibrin clots formed in the absence of calcium or in the presence of FSF inhibitors show a greater susceptibility to fibrinolysis than fibrin clots which are formed in the presence of active FSF (29-31). Gormsen, Fletcher, Alkjaersig, and Sherry found that insoluble clots tended to be more resistant to fibrinolysis than soluble clots but demonstrated only a limited correlation between decreasing clot solubility and resistance to fibrinolysis (31). Most striking was the observation that some insoluble clots were resistant to fibrinolysis while others were just as susceptible as completely soluble clots. Although the methodology used by Gormsen et al. differed from ours, it might be posible to explain their observation on the basis of the studies presented here as well as those reported earlier (10). Fibrin clots become insoluble when dimerization of $\gamma$-chains is complete (Fig. 5) ; however, even after the clot becomes insoluble, cross-linking of the $\alpha$-chains continues for about $90 \mathrm{~min}$. It seems likely, therefore, that the extent of cross-linking of the $\alpha$-chain may make the fibrin clot more resistant to plasmin. This hypothesis, which is now being tested (32), may be of clinical interest because some patients with congenital FSF deficiency manifest a characteristic delayed hemorrhage after trauma (33) that could be due to an increased susceptibility of clots to fibrinolysis (29).

The SDS-gel electrophoretic method of fibrin analysis requires extremely small amounts of material and can be readily performed in any laboratory. Besides its usefulness in unequivocally verifying the diagnosis of FSF deficiency, the electrophoretic methods should be of value in the future in determining if all patients with congenital FSF deficiency are completely lacking FSF activity. When gel scanning equipment is available, the amount of FSF in plasma can be quantitated by determining the serial dilutions of test and normal plasma which cross-link $50 \%$ of the $\gamma$-chain. These methods are also potentially useful in detecting abnormalities of fibrin formation (34-36) which might include rapid crosslinking of $\alpha$ - or $\gamma$-chains, a slow, but otherwise normal cross-linking of the chains, a partial cross-linking of either $\alpha$ - or $\gamma$-chains, incorrect cross-linking of the chains or the complete absence of cross-linking. For example, the methods used here should be able to establish with certainty whether the bleeding defect in the family reported by Hampton, Cunningham, and Bird is due to deficient FSF activity as initially proposed (37), or to an abnormal fibrinogen which does not cross-link, as suggested more recently (35). 


\section{ACKNOWLEDGMENTS}

This work was supported by research grants from the National Heart Institute (HE-06400) and National Institute of Neurological Diseases and Stroke (NS-06233), National Institutes of Health and the North Carolina Heart Association.

The authors wish to thank Dr. Bruce M. Schlein and Mrs. Miriam Marshall for their assistance with this study.

\section{REFERENCES}

1. Robbins, K. C. 1944. A study of the conversion of fibrinogen to fibrin. Amer. J. Physiol. 142: 581.

2. Laki, K., and L. Lorand. 1948. On the solubility of fibrin clots. Science (Washington). 108: 280.

3. Loewy, A. G., K. Dunathan, J. A. Gallant, and B. Gardner. 1961. Fibrinase III. Some enzymatic properties. J. Biol. Chem. 236: 2644.

4. Lorand, L., and K. Konishi. 1964. Activation of the fibrin stabilizing factor of plasma by thrombin. Arch. Biochem. Biophys. 105: 58.

5. Lorand, L., J. Downey, T. Gotch, A. Jacobsen, and S. Kokura. 1966. The transpeptidase system which crosslinks fibrin by $\gamma$-glutamyl-e-lysine bonds. Biochem. Biophys. Res. Commun. 25: 629.

6. Pisano, J. J., J. L. Finlayson, and M. P. Peyton. 1968. Cross-link in fibrin polymerized by factor XIII: $\epsilon-(\gamma-$ glutamyl) lysine. Science (Washington). 160: 892.

7. Matacic, S., and A. G. Loewy. 1968. The identification of isopeptide crosslinks in insoluble fibrin. Biochem. Biophys. Res. Commun. 30: 356.

8. Chen, R., and R. F. Doolittle. 1970. Isolation, characterization and location of a donor acceptor unit from cross-linked fibrin. Proc. Nat. Acad. Sci. U.S.A. 66: 472.

9. McKee, P. A., L. A. Rogers, E. Marler, and R. L. Hill. 1966. The subunit polypeptides of human fibrinogen. Arch. Biochem. Biophys. 116: 271.

10. McKee, P. A., P. Mattock, and R. L. Hill. 1970. Subunit structure of human fibrinogen, soluble fibrin and cross-linked insoluble fibrin. Proc. Nat. Acad. Sci. U.S.A. 66: 738.

11. Kazama, M., and R. D. Langdell. 1969. Purification of fibrin stabilizing factor (FSF). Fed. Proc. 28: 746.

12. Loewy, A. G., K. Dunathan, R. Kriel, and H. L. Wolfinger, Jr. 1961. Fibrinase I. Purification of substrate and enzyme. J. Biol. Chem. 236: 2625.

13. Blomback, B., and M. Blomback. 1956. Purification of human and bovine fibrinogen. Ark. Kemi. 10: 415.

14. Swigert, S., J. L. Koppel, and J. H. Olwin. 1963. Selective inactivation of fibrin stabilizing factor contaminant in fibrinogen. Nature (London), 198: 797.

15. Shulman, S. 1953. The fibrin serum factor. Nature (London). 171: 606.

16. Loewy, A. G., and J. T. Edsall. 1954. Studies on the formation of urea-insoluble fibrin. J. Biol. Chem. 211: 829.

17. Barry, A., and J. M. Delâge. 1964. Congenital deficiency of fibrin stabilizing factor. Observation of a new case. New Engl. J. Med. 272 : 943.

18. Tyler, H. M. 1966. A comparative study of the solvents commonly used to detect fibrin stabilisation. Thromb. Diath. Haemorrh. 16: 61 .
19. Weber, K., and M. Osborn. 1969. The reliability of molecular weight determinations by dodecyl sulfatepolyacrylamide gel electrophoresis. J. Biol. Chem. 244: 4406.

20. Dunker, A. K., and R. R. Rueckert. 1960. Observations on molecular weight determinations on polyacrylamide gel. J. Biol. Chem. 244: 5074.

21. Mills, D., and S. Karpatkin. 1970. Heterogeneity of human fibrinogen: possible relation to proteolysis by thrombin and plasmin as studied by SDS-polyacrylamide gel electrophoresis. Biochem. Biophys. Res. Commun. 40: 206.

22. Brummel, M. C., and R. Montgomery. 1970. Acrylamide gel electrophoresis of the S-sulfo derivatives of fibrinogen. Anal. Biochem. 33: 28.

23. Carter, J. R. 1956. Amperometric titration of $\mathrm{SH}$ groups in purified clotting factors by improved method. Proc. Soc. Exp. Biol. Med. 91: 406.

24. Ellman, G. L. 1959. Tissue sulfhydryl groups. Arch. Biochem. Biophys. 82: 70.

25. Riordan, J. F., and B. L. Valee. 1967. Reactions with Nethylmaleimide and p-mercuribenzoate. Methods Enzymol. $11: 541$.

26. Tyler, H. M., and C. H. Lack. 1964. A tissue fibrin stabilizing factor and fibrinolytic inhibition. Nature (London). 202: 1114.

27 Duckert, F., E. Jung, and D. H. Shmerling. 1960. A hitherto undescribed congenital haemorrhagic diathesis probably due to fibrin stabilizing factor deficiency. Thromb. Diath. Haemorrh. 5: 179.

28. Britten, A. F. H. 1967. Congenital deficiency of factor 13 (fibrin stabilizing factor): report of a case and review of the literature. Amer. J. Med. 43: 751.

29. Bickford, A. F., Jr., and M. Sokolow. 1961. Fibrinolysis as related to the urea solubility of fibrin. Thromb. Diath. Haemorrh. 5 : 480.

30. Lorand, J. B., T. R. E. Pilkington, and L. Lorand. 1966. Inhibitors of fibrin cross-linking: relevance for thrombolysis. Nature (London). 210: 1273.

31. Gormsen, J., A. P. Fletcher, N. Alkjaersig, and S. Sherry. 1967. Enzymic lysis of plasma clots: the influence of fibrin stabilization on lysis rates. Arch. Biochem. Biophys. 120: 654 .

32. Pizzo, S. V., M. L. Schwartz, P. Mattock, R. L. Hill, and P. A. McKee. 1970. Correlation of clottability of purified human fibrinogen with its digestion by purified human plasmin. J. Lab. Clin. Med. 76: 892.

33. Lewis, J. H., D. C. Curiel, W. L. Bayer, and I. L. Szeto. 1970. Factor XIII deficiency: current status. In Hemophilia and New Hemorrhagic States; international symposium, New York. K. M. Brinkhous, editor. The University of North Carolina Press, Chapel Hill, N. C. 257.

34. Egeberg, O. 1967. Inherited fibrinogen abnormality causing thrombophilia. Thromb. Diath. Haemorrh. 17: 176.

35. Hampton, J. W. 1970. Fibrinogen abnormality with defective cross-linkage. Fed. Proc. 29: 647.

36. Jackson, D. P., and E. A. Beck. 1970. Inherited abnormal fibrinogens. In Hemophilia and New Hemorrhagic States; international symposium, New York. K. M. Brinkhous, editor. The University of North Carolina Press, Chapel Hill, N. C. 225.

37. Hampton, J. W., G. R. Cunningham, and R. M. Bird. 1966. The pattern of inheritance of defective fibrinase (factor XIII.). J. Lab. Clin. Med. 67: 914. 